\author{
Military Technical College \\ Kobry El-Kobbah, \\ Cairo, Egypt
}

\author{
$9^{\text {th }}$ International Conference \\ on Civil and Architecture \\ Engineering \\ ICCAE-9-2012
}

\title{
A NEW APPROACH TO INTRPRET DISINTEGRATION, COMPRESSIVE STRENGTH, AND WATER ABSORBTION OF CONCRETE UNDER THE APPLICA TION OF ADMIXTURES
}

Ahmed Mohammed Abdelalim*

\begin{abstract}
This part of research program investigates the influence of applying the widely used additives, such as accelerators (Calcium Chlorides $\mathrm{CaCl}_{2}$ ), Inhibitors (Sodium Nitrite $\mathrm{NaNO}_{2}$ ), and Water Reducing Agents (Super-Plasticizer) on the Mechanical properties of concrete; The Uniaxial Compressive Strength, Absorption of water, and Disintegration of concrete at different time intervals, to introduce an imagination of concrete behavior when chemicals are used. These additives were varyingly added to plain concrete cube samples. Meanwhile control mixes out of the interference of chemicals were prepared simultaneously, to deduce a rational conception about the best behaving mix against harmful surrounding conditions. A mathematical treatise was discussed from the experimental data to interpolate the results of each category for each test.

The essence of this research surpasses the straightforward announcement for the advantages of additives to a balanced comparison between benefits gained and the undesirable sequences resulted from .It has been found that the use of certain substances inevitably brings problems to concrete durability against aggressive circumstances (results indicate that mixes contain sulfates fail quickly under the disintegration of concrete test despite the prior strength oftenly noticed). The use of aim-contradicting subjects gives high resistibility against corrosion of reinforcement [1]. But it doesn't commensurate with the damage of corrosion (disintegration) of concrete itself. So, results as well as mathematical manipulations give us an evaluation of how additives act and a prediction of their future effect on concrete.
\end{abstract}

Keywords: Accelerators, Inhibitors, Water-Reducing Agent, Uniaxial Compressive strength, Disintegration, Absorption, Mathematical Manipulation.

\section{INTRODUCTION}

The use of additives has grown up parallely with the revolutionary changes in concrete technology nowadays. The first signs of imparting additiv es in concrete manufacture returns back to the durable repairing trials of RC structures built towards

* Lecturer of RC Structures, CSC Academy, Cairo, Egypt. 
the end of the nineteenth century and the early part of the twentieth. After then, the purpose of producing certain chemical compounds can effectively be used for imparting some additional and desirable characteristic(s).

In fact, this research doesn't review for the previous works, neither emphasizing post proved results. Whereas, it inspired the major trend of the last researches results, concluded them and built upon. It concentrates on the influence of the most widely used additives on the corrosion of embedded reinforcement pre-discussed in the first part [1], in additional to the mechanical properties of resulted concretes . The two parts integrates together to cover both chemical and mechanical properties of concrete when additives used.

\section{EXPERIMENTAL WORK}

Developing an obvious imagination of how additives influence the mechanical properties necessitates keeping the same proportioning adopted before. So, Calcium Chloride $\left(\mathrm{CaCl}_{2}\right)$ as an accelerator, Sodium Nitrite $\left(\mathrm{NaNO}_{2}\right)$ as inhibitor, Sodium Sulfate $\left(\mathrm{Na}_{2} \mathrm{SO}_{4}\right)$ as an accelerator and a source of sulfates, and Super-Plasticizer (here and after denoted with S.P) as a Water-Reducing Agent were the chemicals used. Ordinary Portland cement, natural sand and gravel (whose sieve analysis is shown in tables (1), (2). The mix proportions were (1: 2: 4, cement: sand: gravel), with a $400 \mathrm{Kg} / \mathrm{m}^{3}$ cement content. A water/cement ratio (0.4) was maintained constant to fabricate all concrete specimen ( cubes $5,7,15 \mathrm{~cm}$ ). Additives were varyingly added to plain concrete samples (table 3). The corresponding Uniaxial Compressive Strength, Water Absorption, and Disintegration (Corrosion) of Concrete were all determined for different mixes at different time intervals, to conclude finally the advantages and disadvantages of these additives and determine each of which was the best behaving mix.

Table (1): Sieve analysis of natural sand.

\begin{tabular}{|c||c||c|c||c||c||c|}
\hline Sieve No. & $\mathbf{5}$ & $\mathbf{7}$ & $\mathbf{1 4}$ & $\mathbf{2 5}$ & $\mathbf{5 0}$ & $\mathbf{1 0 0}$ \\
\hline \hline $\begin{array}{c}\text { \%age } \\
\text { Passing }\end{array}$ & 97.2 & 93.2 & 77.2 & 46.3 & 11.2 & 0.9 \\
\hline
\end{tabular}

Table (2): Sieve analysis of gravel.

\begin{tabular}{|l||c||c||c||c||c|}
\hline Sieve No. & $\mathbf{4 0}$ & $\mathbf{2 0}$ & $\mathbf{1 0}$ & $\mathbf{5}$ & Pan \\
\hline \hline \% passing. & 98.78 & 61.94 & 19.79 & 2.82 & 0 \\
\hline
\end{tabular}


Table (3): Description of mixes.

\begin{tabular}{|c||c||c||c||c||c||c||}
\hline ADDIIVES & W/C & $\begin{array}{c}\text { MIX } \\
\text { NO(1) }\end{array}$ & $\begin{array}{c}\text { MIX } \\
\text { NO(2) }\end{array}$ & $\begin{array}{c}\text { MIX } \\
\text { NO(3) }\end{array}$ & $\begin{array}{c}\text { MIX } \\
\text { NO(4) }\end{array}$ & $\begin{array}{c}\text { MIX } \\
\text { NO(5) }\end{array}$ \\
\hline \hline $\mathbf{S . P}$ & 0.4 & $1 \%$ & $1 \%$ & $1 \%$ & $1 \%$ & $1 \%$ \\
\hline \hline $\mathbf{C a C l}_{2}$ & 0.4 & ----- & $0.5 \%$ & $0.5 \%$ & $0.5 \%$ & $0.5 \%$ \\
\hline \hline $\mathbf{N a C l}$ & 0.4 & ---- & $0.5 \%$ & $0.5 \%$ & $0.5 \%$ & $0.5 \%$ \\
\hline \hline $\mathbf{N a}_{2} \mathbf{S}_{4}$ & 0.4 & ---- & ---- & $1 \%$ & $1 \%$ & $-\cdot--$ \\
\hline \hline $\mathbf{N a N O}_{2}$ & 0.4 & ----- & ---- & ---- & $1 \%$ & $1 \%$ \\
\hline
\end{tabular}

Choices adopted in this research are referred to many considerations:

First, at least one mix should be kept out of the interference of chemicals for comparing purposes, to deduce either the use of additives is less desira ble or not using. The main categories of admixtures were used in research, as (S.P) represents the (Water-Reducing-Agent), $\left(\mathrm{CaCl}_{2} \& \mathrm{Na}_{2} \mathrm{SO}_{4}\right)$ as Accelerators, while ( $\left.\mathrm{NaNO}_{2}\right)$ represents Inhibitors .The w/c ratio and percentage of S.P were kept constant for all mixes to ensure the same workability. Similarly, $\mathrm{NaCl}$ is kept constant for measuring corrosion of steel electrochemically.

For Mix No.(2): $\mathrm{CaCl}_{2}$ was used as an accelerator to increase prior strength and as a resource of Chlorine ions $\left(\mathrm{Cl}^{-}\right)$to ensure the occurrence of corrosion.

For Mix No.(3): the presence of $\left(\mathrm{CaCl}_{2} \& \mathrm{Na}_{2} \mathrm{SO}_{4}\right)$ simultaneously intensify the influence of corrosive environment, $\mathrm{Na}_{2} \mathrm{SO}_{4}$ also acts as a catalyst in cor rosion of concrete due to the presence of sulfate element.

For Mix No. (4): $\mathrm{NaNO}_{2}$ as Inhibitor hinders the dual effect of $\mathrm{CaCl}_{2}$ and $\mathrm{Na}_{2} \mathrm{SO}_{4}$ in corrosion of steel.

For Mix No.(5): the absence of $\mathrm{Na}_{2} \mathrm{SO}_{4}$ reduces the corrosion of steel reinforcement. This classification indicates why these additives in particular were chosen.

\section{WATER ABSORPTION TEST}

Cubes of size $(15 \times 15 \times 15 \mathrm{~cm})$ were dried after demoulding in an oven for 24 hours and then weighed. Weights were recorded to indicate the weight of cubes before absorption. Cubes were soaked in water for different time intervals $(5,10,15,20,30,60$ minutes) and (1,1.5,24,48 hours). Then, weights after absorption were determined at the previous time intervals. The percentage of absorption is calculated as the percentage of weight increase referred to the original weight.

[Percentage of Absorption = weight after absorption - weight before absorption /

Weight before absorption].

The Shieken Parameters of Water Absorption adopted here, denoted with "Dm" and " $\mathrm{H}$ " [3]. These parameters were determined using the following equations :

$$
\mathrm{H}=\ln \left[\ln \left(1-\mathrm{Wt}_{2} / \mathrm{Wt}_{\max }\right) / \ln \left(1-\mathrm{Wt}_{1} / \mathrm{Wt}_{\max }\right)\right] /\left(\ln \left(\mathrm{t}_{2} / \mathrm{t}_{1}\right)\right.
$$




$$
\left.\mathrm{Dm}=\ln \left(\mathrm{Wt}_{\max } / \mathrm{Wt}_{\max }-\mathrm{Wt}_{1}\right) / \mathrm{t}_{1}{ }^{H}\right)
$$

Where:

$\mathrm{H}$ : describes the homogeneity of pores, Dm: describes the main diameter of pores,

$\mathrm{W}_{\max }$ : the max \%age of water absorbed, $\mathrm{Wt}_{1}$ : the \%age of water absorbed after time $\mathrm{t}_{1}$

$\mathrm{Wt}_{2}$ : the \%age of water absorbed after time $\mathrm{t}_{2}$, and $\mathrm{t}_{1, \mathrm{t} 2}$ time intervals ( hours).

\section{DISINTEGRATION OF CONCRETE}

Cubes $(15 \times 15 \times 15 \mathrm{~cm})$ which precast before, were dried in an oven at $\left(105^{\circ}\right)$ temperature for 24 hours. After drying, each cube was weighed and its weight was recorded .On the other hand, a solution of (5\% by weight) of Sodium Sulfate $\left(\mathrm{Na}_{2} \mathrm{SO}_{4}\right)$ was prepared. Then cubes were soaked in the solution before -prepared in a manner insuring that the surface of solution covers all cubes. Cubes wee observed regularly and solution was changed every 15 days to keep the same concentration. They still in solution for 35 days then weighed again and the new weights were recorded. A comparison was made between the original weights of cubes before putting it in solution. After then, the loss of weight is observed due to the corrosion of concrete. A new term "The Rate of Disintegration (Corrosion of Concrete)" was introduced as the percentage of loss of weight referred to the original weight. The Rate of Disintegration can be expressed mathematically as:

[Percentage of Disintegration $=$ Loss of weight $/$ original weight $]$

\section{UNIAXIAL COMPRESSIVE STRENGTH OF CONCRETE}

The Cube-Crushing Method was adopted here as the laboratory test of Compressive Strength (according to ASTM: C 349-82). Six cubes of each mix were made, with the passage of $1,3,7,28$ days they were crushed to observe the growth of strength cyclically.

\section{RESULTS AND DISCUSSION}

\section{6-1. Discussion of Absorption Test}

The control mix has a relatively high absorption percentage after 5 minutes compared with other mixes reflecting a high absorptive tendency. This is may be due to the change of the shape of mixes contain chemicals. The volume of pores may change too; changes in pores shape and volume differ from mix to another inspite of fixing all ambient conditions (curing, temperature, etc...). For control mix , "Dm" approaches its maximum value which reflects the increment of main diameter of pores, and the percentage of absorption consequently, this is may be due to that chemical substances increase the hydration process of cement which increases the diffusion of cement in $\mathrm{m}$ Mix No.2 $\left(0.5 \% \mathrm{CaCl}_{2}\right)$ has the lowest value of "Dm" and the highest of " $\mathrm{H}$ ", which 
indicates that homogeneity of pores in mix No. 2 was the best, their volumes were the smallest (Low absorptive tendency). So, generally the presence of chemicals reduces the tendency of absorption. (Figs. 1, 2, 3, 4, 5, 6).

\section{6-2. Discussion of Disintegration of Concrete}

Disintegration was mainly detected by loss of weight. A comparis on was made on the corresponding compressive strength to deduce the harmful effect of the aggressive medium (aqueous $\mathrm{Na}_{2} \mathrm{SO}_{4}$ ). It has been found that mixes contain $\mathrm{CaCl}_{2}$ resists disintegration of concrete, with a small loss of weight and change of final strength, (figs.7,8). Mixes contain $\mathrm{Na}_{2} \mathrm{SO}_{4}$ have a low resistibility to corrosion (disintegration) of concrete and the final strength decreased obviously. Mix No.5 was the best behaving mix against disintegration through containing aim -contradicting additives (accelerator and inhibitor). Mix No.4 fails quickly under disintegration test, not only for the dual effect of accelerators but also the high corrosive medium caus es a reduction in the $\mathrm{PH}$ value in the surrounding concrete reducing concrete resistivity [4]. Another explanation may be convincingly introduced, the abundance of $\left(\mathrm{H}_{2} \mathrm{~S}\right)$ in

the aggressive medium which interacts with calcium in cement form ing Calcium Sulfates $\left(\mathrm{Ca}_{2} \mathrm{SO}_{4}\right) .\left(\mathrm{Ca}_{2} \mathrm{SO}_{4}\right)$ attacks the surface of concrete aggressively turning it to a wet, porous, scoured, weak, easy to be spilled, tend to be yellowish layer. [ 5]

\section{6-3. Brief Discussion of Compressive Strength Test}

It has been observed throughout tests that the Control Mix (mix No.1) has a relatively low prior strength compared with those contain chemical substances (Calcium chloride and Sodium Sulfate in particular), (fig.9-a,b) . Meanwhile, mixes contain accelerators $\left(\mathrm{CaCl}_{2}\right.$ and $\left.\mathrm{Na}_{2} \mathrm{SO}_{4}\right)$ have high final strength, beside that other mixes have slight differences between their final strength. But, even the p resence of accelerators of great influence on the gain of prior strength, but on the other hand the increment decreases gradually with the passage of time (as noticed after 35 days).

\section{MATHEMATICAL MAINPULATION}

When tests on the mechanical properties were operated, results were tabulated and presented too. The shape of curves plotted led to the trial of expressing them mathematically, so curve fitting was the second step. Thus, a curve fitting of experimental results based on the values of least-squares method was operated to conclude a mathematical expression satisfies the major trend of each mix under each test. This technique has a dual beneficial gain, partly as it interpret the physical phenomenon results ascertaining them strongly, otherwise it h elps in predicting the future behavior of this mix without needing experiments any further, and save much more time and effort. This only occurs when the coincidence of resulted remarks and plotted curve fitted preciously.

\section{7-1. Curve Fitting of Absorption Test}

For Control Mix, the fitting curve $\left[\mathrm{Y}=3.022 \mathrm{e}^{\wedge}\left[(\operatorname{LnX}-8.949)^{\wedge} 2 /-35.58\right] \ldots \ldots . . . . .(3)\right.$ 
For Mix No.(2), [ $\mathrm{Y}=-0.3925+0.3883 * \operatorname{Ln} \mathrm{X}]$

For Mix No.(3), $[\mathrm{Y}=0.02693+0.2797 * \operatorname{Ln} \mathrm{X}]$

For Mix No. (4), $\left[\mathrm{Y}=0.6579 *(0.1394) \wedge(1 / \mathrm{X}) * \mathrm{X}^{\wedge}(0.1533) \ldots\right] \ldots \ldots .$.

For Mix No. (5), [Y $=0.1411+0.2813 * \operatorname{Ln} \mathrm{X}]$

Or, $\left[\mathrm{Y}=0.5495 * \mathrm{X}^{(0.1932)}\right.$

Where: (Y: the percentage of water absorption as a gain in weight after time $\mathrm{T}$ )

$:(\mathrm{T}:$ time in minutes).

As remarked from the curve fitting, equations commensurate with the basic concept of water absorption, i.e., the logarithmic function " $L n$ " representing that a slight "Y" gain for great values of " $\mathrm{X}$ ". Now, referring to the main conception of critical points and their indications within the behavior of different mixes .Considering water absorption test, these peak points represent approaching the full-saturation state. At certain time pores were theoretically saturated, absorbing no more water. This hypothesis require enhancement both experimentally and mathematically. First, mathematically can be proved regarding Mix No.1 (control mix).

For Mix No.1, the fitted curve $\left[\mathrm{Y}=3.022 * \mathrm{e}^{\left.(\ln X-8.8949)^{\frac{2}{1-35.58}}\right]}\right.$

$$
\begin{aligned}
\mathrm{Y}^{\prime}=-71.16 \mathrm{e}^{\wedge}\left\{\frac{\left[35.58 /(\ln X-8.949)^{2}\right] \times(\ln X-8.949)(1 / X)}{(\ln X-8.949)^{4}}\right\} & \frac{35.58}{(\ln X-8.949)^{2}}
\end{aligned}
$$

So, the critical points exist for values of $\mathrm{X}$ verify the following:

$$
\begin{gathered}
(\ln X-8.949)=0 \quad \Leftrightarrow \quad Y^{\prime}=\infty \\
(1 / X)=0 \quad \Leftrightarrow \quad X=\infty \\
\text { So, for }(\ln X-8.949)=0 \quad \Rightarrow X=7700 \text { min }==5.34 \text { days. }
\end{gathered}
$$

Comparing the last derived result with experimental data, they more or less coincident to each other, as $X=(6680 \mathrm{~min}$. $)$ represents the peak point of curve fitted before ; readings after then begin to reduce gradually. If this value in particular was applied in "Dm", "H" laws (Eq.1\&2), we see how it makes "Dm" approaches its minimum value, whereas " $H$ " approaches its maximum value. (i.e., the smallest main diameter of pores at the most homogeneous state of these pores, as they inversely proportional to each other).This logic assumption necessitates emphasis. To do so, the relationship between the homogeneity and the main diameter of pores was plotted to derive a mathematical expression of " $\mathrm{H}$ " as a function of "Dm". This was found;

$$
\left[\mathrm{H}=\left(0.8241 \times 10^{7}\right)\left(0.1659 \times 10^{-7}\right)^{(1 / D m)} * \frac{1}{D m^{24.85}}\right] \ldots \ldots \ldots
$$

Thus, the inverse proportionality before-noticed, demonstrates that the homogeneity of mix in general decreases with the increment of main diameter of po res. Applying the same technique on other mixes can lead to a rational understanding of their ability to absorb water, and to anyhow.

\section{7-2. Curve Fitting of Disintegration (CORROSION of CONCRETE)}


Results of disintegration detected with respect to lo ss of weight were all interpolated for all mixes, the weight after disintegration as a function of original weight was fitted. For mix No.1, the fitting equation: $[\mathrm{Y}=-1263+273.3 \ln \mathrm{X}]$

For Mix No.2, the fitting equation: $[\mathrm{Y}=1 /(0.02719-0.004185 \ln \mathrm{X})]$.

For Mix No.3, $\left[\mathrm{Y}=1 /\left\{0.0000004883(\mathrm{X}-283.1)^{2}+0.003672\right\}\right]$.

For Mix No.4, the fitting equation, $[\mathrm{Y}=-1704+352.1 \ln \mathrm{X}]$

For Mix No.5, the fitting equation, $[\mathrm{Y}=1 /(0.02046-0.002991 \ln \mathrm{X})]$

Where: Y; weight of standard cube after disintegration in grams.

, $\mathrm{X}$; time in minutes.

Equations verify the greater the value of (Y) represents the lower tendency to lose weight after disintegration which reflects a rising resistibility against disintegration.

The previous equations illustrate the behavior of each mix. Mixes No. (2\&5) give the highest values of $(\mathrm{Y})$ with increasing values of $(\mathrm{X})$, i.e., they exhibit high resistance against disintegration. Comparing their attitudes in particular, both represented by a fractional function of the same nominator, but the denominator of Mix No.5 is smaller than that of Mix No.2. This strengthens the conclusion of better behaving attitude of accelerator $\left(\mathrm{CaCl}_{2}\right)$ and Inhibitor $\left(\mathrm{NaNO}_{2}\right)$ against disintegration. This may be explained as the presence of this accelerator reduces the main diameter of pores, reducing its absorptive tendency which accompanied with the inhibitor effect against harmful sulfates and finally resists disintegration. Otherwise, Mixes $(1,2,3)$ of the fitted equations $(9,11,12)$ explain how the major trend of them all seems to be alike . Where a mix without chemicals (equation 9) has a less subtracted value with a larger multiplier constant to the $2^{\text {nd }}$ term compared with (equation. 12) verifying the better behavior of Mix No.(1) against disintegration. The greater constant value of Mix No.3 may reflects its high initial response to disintegration due to the presence of sulf ates actually in $\left(\mathrm{Na}_{2} \mathrm{SO}_{4}\right)$. This is applicable also for Mix No.3 where the fractional function with an increasing value of denominator with increment of $(\mathrm{X})$. Thus, the greater values of $(\mathrm{X})$, the lower values of $(\mathrm{Y})$, i.e ., the greater the influence of harmful surrounding media (aggressive disintegrative aqueous of $\left(\mathrm{CaCl}{ }_{2}, \mathrm{Na}_{2} \mathrm{SO}_{4}\right)$. The configuration of equations with expected behavior of different mixes intensify the demand for mathematical manipulations.

\section{7-3: Curve Fitting of Compressive Strength Test}

Relations studied the strength as a function of time;

For Mix No.1, the best fitting curve: $\left[\mathrm{Y}=319.7-1369 / \mathrm{X}+1858 / \mathrm{X}^{2}\right]$.

For Mix No.2, the fitting curve: $\quad\left[\mathrm{Y}=328.3-621.4 / \mathrm{X}+366.6 / \mathrm{X}^{2}\right]$

For Mix No.3, the fitting curve: $\left[\mathrm{Y}=93.54^{*}(0.994)^{x} *(\mathrm{X})^{0.387}\right] \ldots \ldots \ldots$.

For Mix No.4, the fitting curve: $[\mathrm{Y}=256.7+0.9912 * \mathrm{X}-389.3 / \mathrm{X}]$

For Mix No.5, the fitting curve: $\left[Y=353.3-1449 / X+1901 / \mathrm{X}^{2}\right]$.

Where: (compressive strength after time $\mathrm{X}$ in days).

From these fitting curves, it can be concluded that almost all mixes have the trend of "Parabolic Function" with varying constant value differs from a m ix to another. The $14^{\text {th }}$ equation has the smallest coefficient of $\left(\mathrm{X}^{2}\right)$ with a slight gain in $(\mathrm{Y})$ values With the passage of time, it decreases gradually for the long run as geometrically plotted by an ascending half of a parabola (quadrat ic function). The major trend of all mixes 
seemed to be the same with a different initiative response, ended normally to the neighborhood of a straight inclined line intersects with the parabola at infinity representing the endest value (gain) of related p roperty (at the very later time). The lowest-plotted curve of control mix explained its lower strengths compared with those where additives used. The $15^{\text {th }}$ equation demonstrates the effect of accelerators, which seen clearly as the relatively high constant value, increasing coefficient of $\left(\mathrm{X}^{2}\right)$, and lastly the highest-plotted curve commensurates with prior inspections. For higher values of $\mathrm{X}$ (time), the describency tends to disappear. The same remarks can be noticed for equations ( $16 \& 17$ ) with a higher gain in the very early ages which may be due to the presence of two accelerating agents. For Mix No (5) [equation 18], the curve nearly coincided with that of Mix No. (2), which means that Mix No.5 gives rewarding results of strength with ti me beside its high resistibility against corrosion of reinforcing steel $[6,7]$. Predictions carried out due to the mathematical analysis indicates that the percentages of gain in compressive strength for all mixes after 90days, a year, and three years more or less do not exceed about $1.2 \%$ of the $28^{\text {th }}$ compressive strength (ignoring other ambient conditions that cause increment).To prove that differences tend to disappear at latest ages, the neighboring approximate lines to fitted curves were plotted and thei $\mathrm{r}$ slopes were all determined at infinity. So, more or less no real difference in slopes which indicates that even these mixes differ in their prior strength they may have the same strength with the long run.

\section{CONCLUSIONS}

1. While Mixes contain accelerator s tends to gain greater compressive strength up to 28 days compared with those contain inhibitors or neither have chemicals at all , but later on the growth of strength varies slightly and variance reduces with the passage of time.

2. Using additives changes the interior structure of pores inside concrete; it reduces their main diameter and the absorptive tendency consequently.

3. Mix No.2 has the lowest absorptive tendency, this may be due to increasing the hydration process of cement which increases its diffusi on in mix and the smaller the main diameter of pores resulted from.

4. Mix No.4, which contains $\left(\mathrm{CaCl}_{2}, \mathrm{Na}_{2} \mathrm{SO}_{4}\right)$ has the lowest resistibility against disintegration which assures that aggregates contain sulfates must not used in concretes exposed to aggressive media.

5. Mix No.5, including an $\left(0.5 \% \mathrm{CaCl}_{2}\right.$ as an accelerator, and $1 \% \mathrm{NaNO}_{2}$ as inhibitor) was the best behaving mix against disintegration, has a low absorptive tendency, and has the highest final strength.

6. The lower the main diameter of pores the higher the homogeneity of mix.

7. The control mix even exhibits high prior absorptive tendency but approaches the same degree of absorption after several days (pre -determined in mathematical discussions) may be attained using additives.

\section{RECOMMENDATIONS}


As every thing changes, nothing remains constant. Changes occur along intervals. So, the rate of change can be detected, the limit of this rate can be estimated. Studying the characteristics of plotted curves can give a precious evaluation of the studied property throughout the points of inflection, the continuity of function, and the major trend, etc. So, much more consideration of mathematical manipulations is preferably recommended.

\section{REFERENCES}

[1] Ahmed Mohammed Abdelalim and A. E .Sayed," The effect of additives on the corrosion of reinforcing steel in concrete", Part.1. international Conference on Material Science and Technology, April 2-4,2001, Beni-Suief ,Egypt. [2]Ravindarajahn and R.Ong, "Corrosion of steel in concrete in relation to bar diameter and cover thickness. Concrete durability, American concrete institute, 1987, pp. 100-112.

[3] Mamdouh M. Nassar, “corrosion prevention and Control” , 1997, pp. 167-172.

[4] H. L. Heys, "Physical Chemistry", $5^{\text {th }}$ edition, Harrap, London. p. 393.

[5] M. Raupach, "Chloride - induced microcell corrosion of steel in concrete", Aachen, Technische Hochschule, Fachbereich 3, Dissertation 1992.

[6] R.D. Browne, "Mechanisms of corrosion of steel in concrete in relation to design , inspection and repair of offshore and coastal structures. Performance of concrete in marine environment, American Concrete Institute, pp. 65, 169.

[7] M.I.Mos, K.G. Babu, and H.H Asker" .Electrolytic Accelerated Corrosion Cracking_Studies”, ICCE-1, 24-26 March 1998, Cairo, PP. 671-687. 


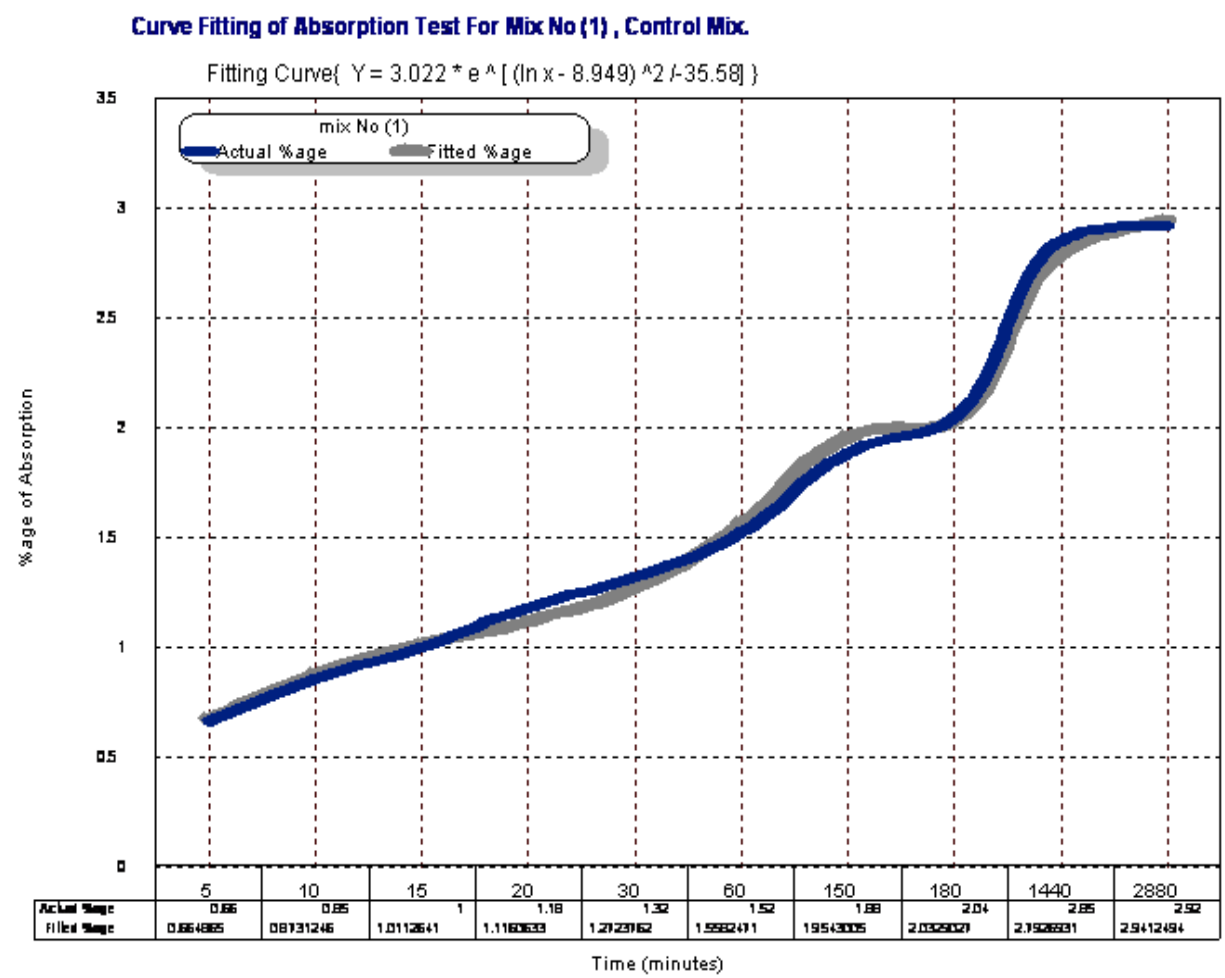

Gurve fitting of Mix No (2), Absorption test.

Equation : $[Y=-0.3925+0.3883 * \operatorname{Ln} \times]$

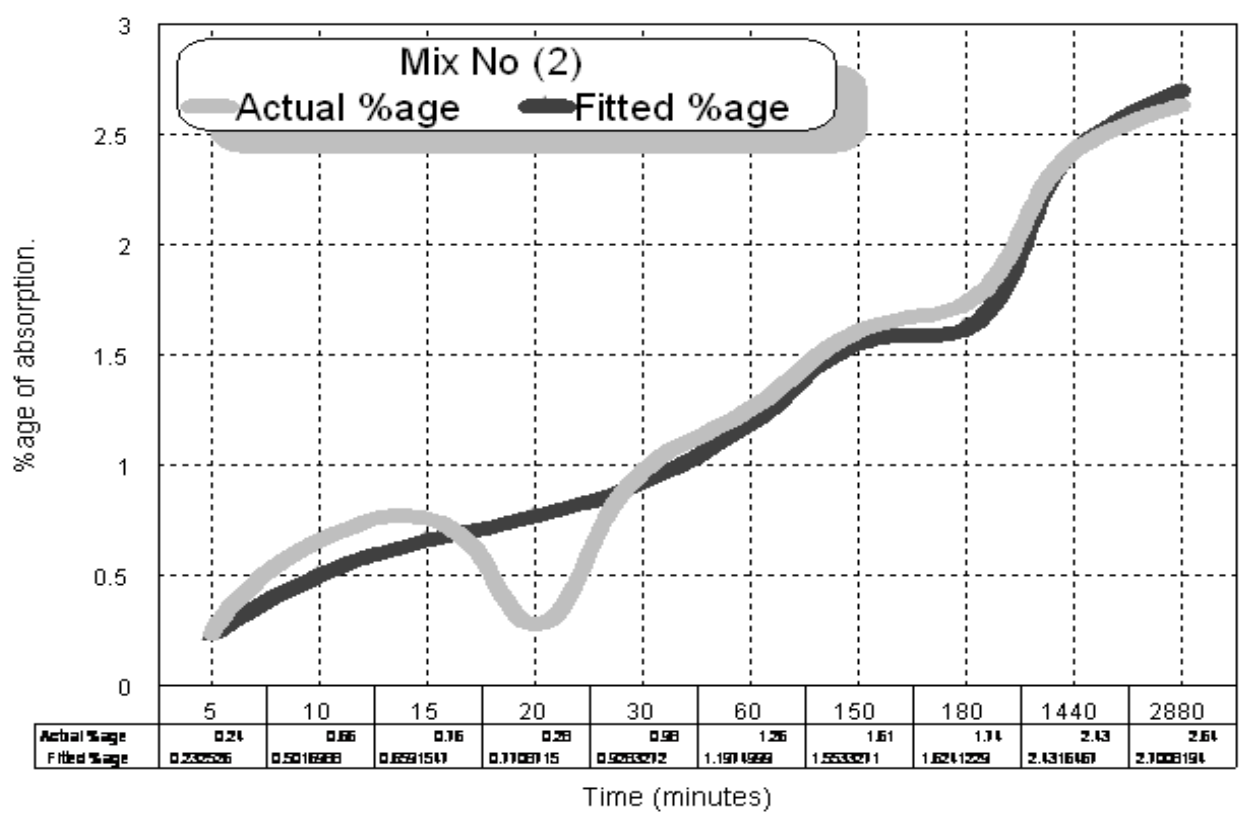

Fig.1, 2: Absorption test results for Mix 1, 2. 

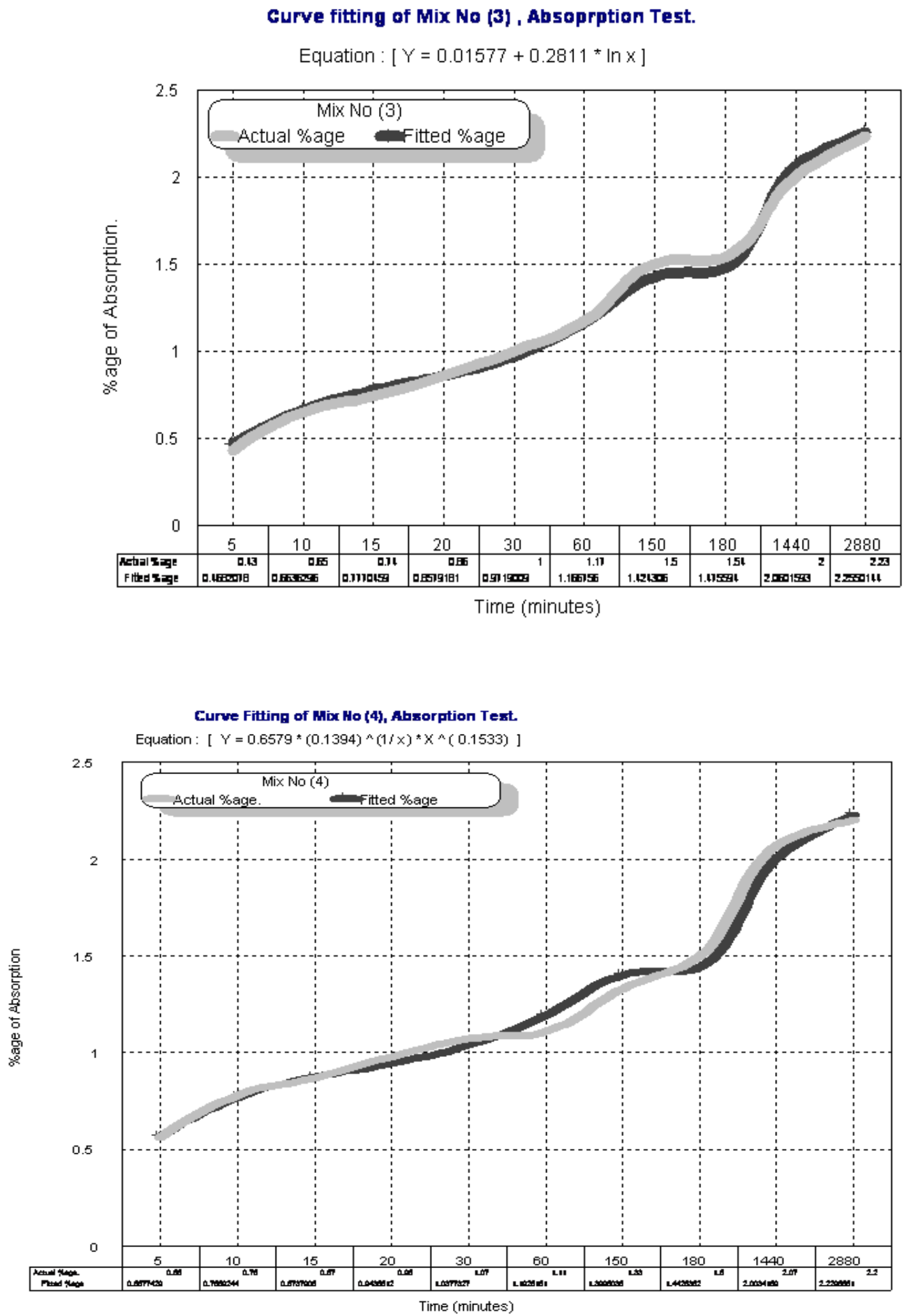

Fig.3, 4: Absorption test results for Mix 3, 4. 


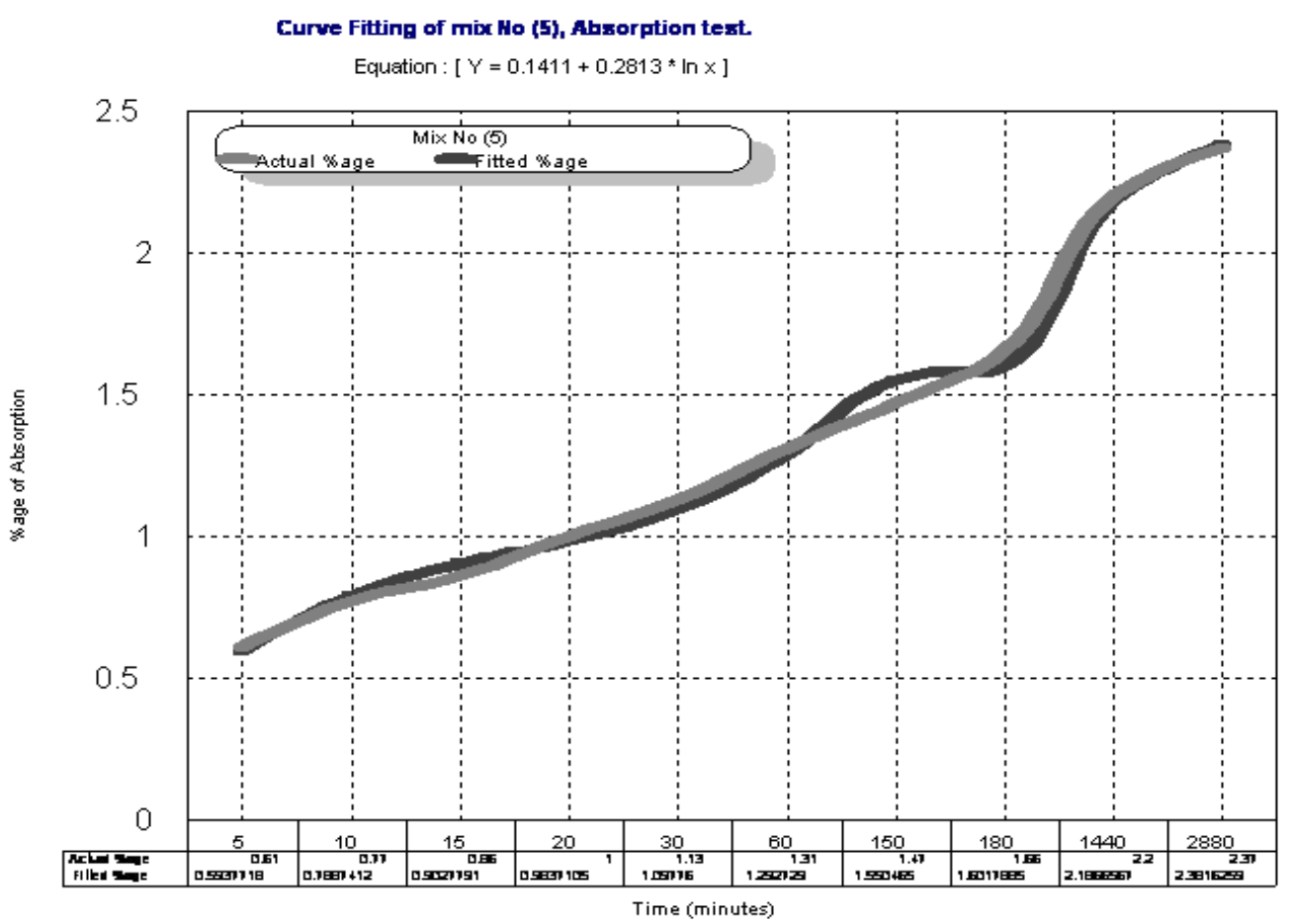

Reswlts of Water Abromtion Test at different Time intenats ( 5, 76, 75, 26 , $30,60,750,780,7446,2880$ minutesi)

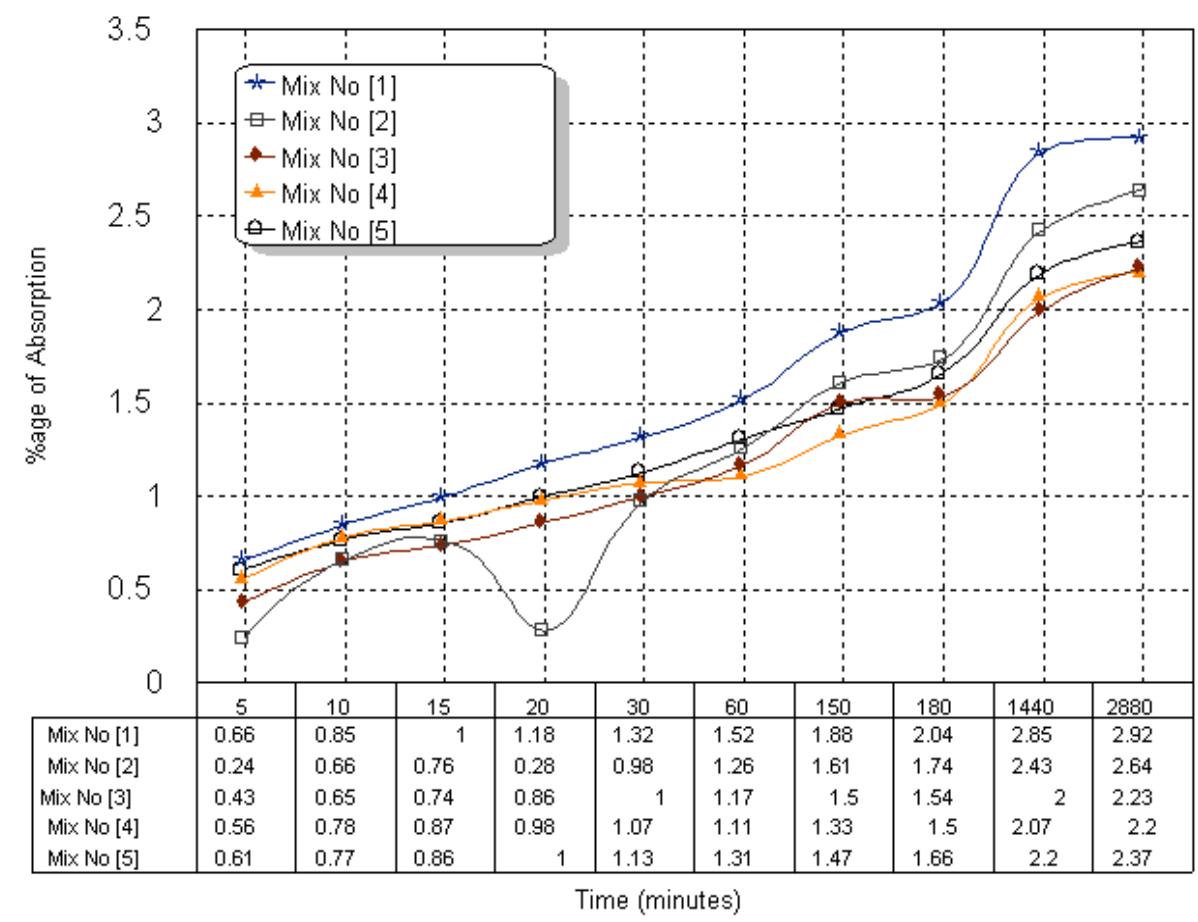

Fig.5, 6: Absorption test results for Mix 5, accumulative for all mixes. 
Fig.(7): Results of Corrosion of Concrete after 35 days ( with Respect to the

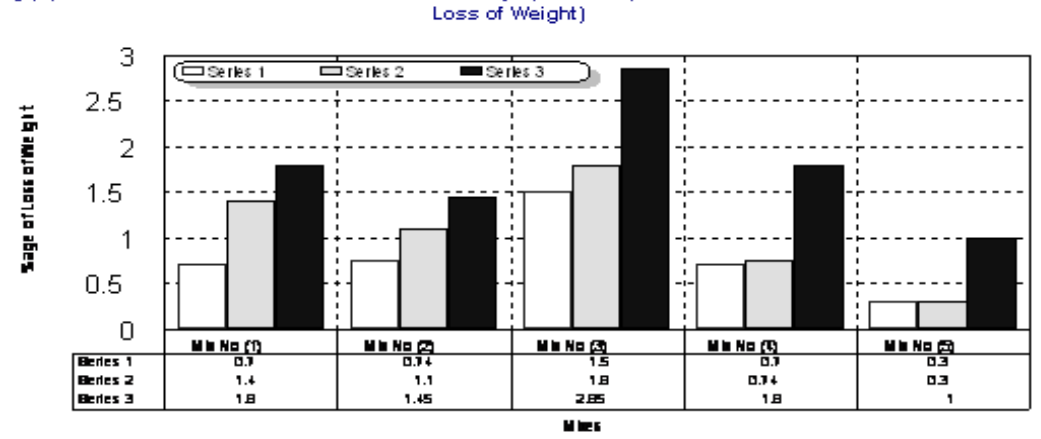

Fig.(8): Results of Disintegration of concrete ( withRespect to the Loss of

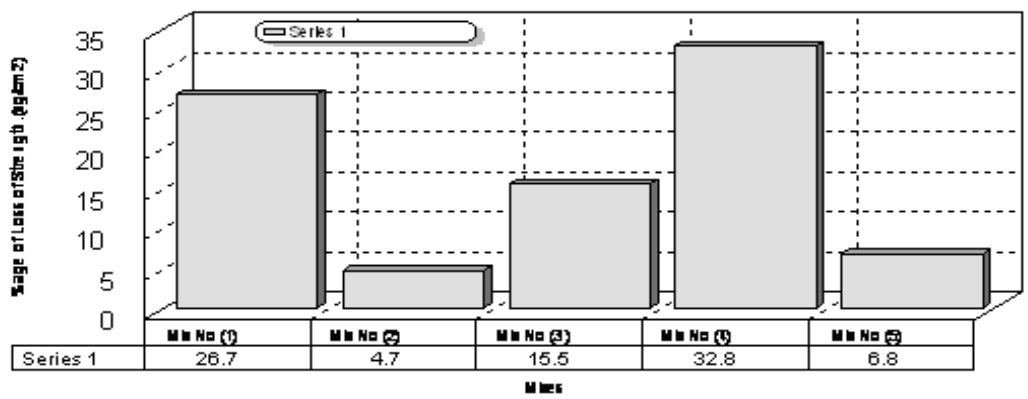

Fig.(9-a): The Growth of Compressive Strength for the Different Five Mixes.

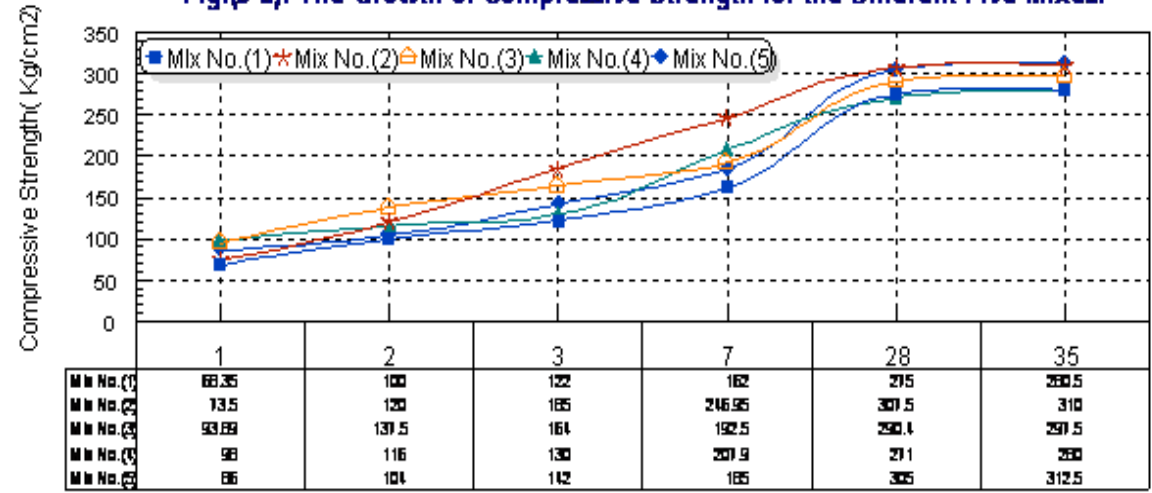

Time (days)

Fig.(9-b):Results of Uniaxial compressive Strength at $7,28,35$ days.

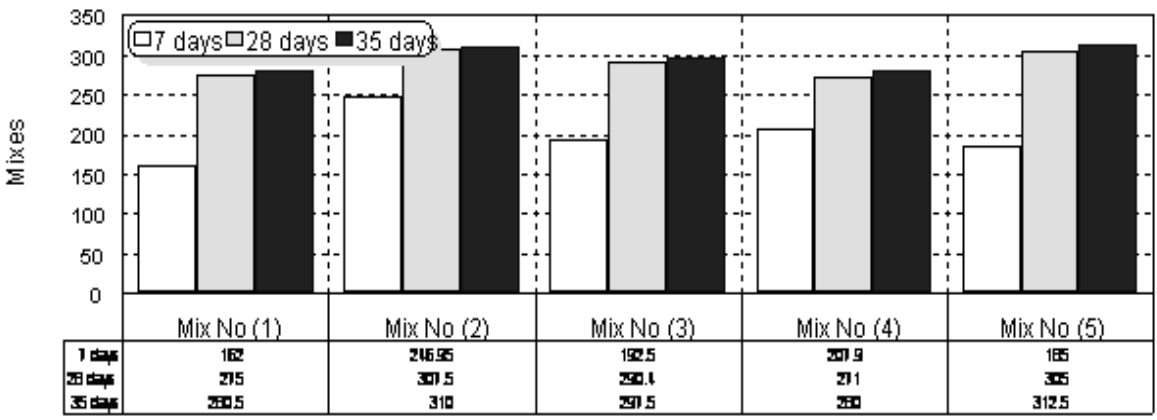

Compressive Strenqth ( $\mathrm{kg} / \mathrm{cm} 2)$ 